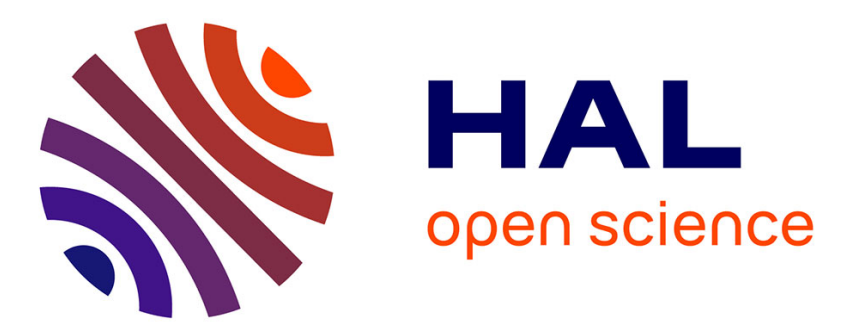

\title{
Spontaneous 2D modulation instability in second harmonic generation process
}

\author{
M. Delqué, G. Fanjoux, S.-P. Gorza, M. Haelterman
}

\section{To cite this version:}

M. Delqué, G. Fanjoux, S.-P. Gorza, M. Haelterman. Spontaneous 2D modulation instability in second harmonic generation process. Optics Communications, 2011, 284 (5), pp.1401-1404. 10.1016/j.optcom.2010.10.098 . hal-00655128

\section{HAL Id: hal-00655128 \\ https://hal.science/hal-00655128}

Submitted on 14 Apr 2021

HAL is a multi-disciplinary open access archive for the deposit and dissemination of scientific research documents, whether they are published or not. The documents may come from teaching and research institutions in France or abroad, or from public or private research centers.
L'archive ouverte pluridisciplinaire HAL, est destinée au dépôt et à la diffusion de documents scientifiques de niveau recherche, publiés ou non, émanant des établissements d'enseignement et de recherche français ou étrangers, des laboratoires publics ou privés. 


\title{
Spontaneous 2D modulation instability in second harmonic generation process
}

\author{
Michaël Delqué $^{\mathrm{a}, \mathrm{b}, *}$, Gil Fanjoux ${ }^{\mathrm{b}}$, Simon-Pierre Gorza ${ }^{\mathrm{a}}$, Marc Haelterman ${ }^{\text {a }}$ \\ a Service OPERA-photonique, CP194/5, Université Libre de Bruxelles U.L.B., Avenue F.D. Roosevelt, 1050 Bruxelles, Belgium \\ b Département d'Optique P.M. Duffieux, Institut FEMTO-ST, Université de Franche-Comté, CNRS UMR 6174, F-25030 Besançon, France
}

Two-dimensional modulation instability (2DMI) is experimentally demonstrated in a classical second harmonic generation setup. The spatial spectrum is measured and reveals typical 2DMI bands, in agreement with the analytical MI model. These observations are confirmed by $(2+1) \mathrm{D}$ numerical simulations.

\section{Introduction}

Since the pioneering work of Torruellas et al. [1], Torner et al. [2,3] and Boardman et al. [4], it is well-known that second-order nonlinearity $\left(\chi^{(2)}\right)$ can lead to efficient spatial effects. Quadratic media commonly used for frequency conversion were then demonstrated to allow for soliton propagation (see ref. [5] for a review), in particular in $(2+1)$ D configuration $[1,3,6]$ where Kerr solitons are not stable [7].

As expected in a medium where solitons can be generated, modulation instability (MI) [8,9] is observable in $\chi^{(2)}$ medium [10]. MI results from the interplay between dispersion (or diffraction) effects and nonlinearity in the case of a field transversely extended in time (or space, respectively). The plane wave solution is no longer stable and breaks up in soliton-like components. This phenomenon widely observed in nonlinear optics is responsible for pulse train generation in optical fibers [11], spatial Kerr filamentation [12] and beam breakup in $\chi^{(2)}$ medium [13]. $\chi^{(2)}$ MI has been extensively studied in transverse 1D, i.e., planar waveguide [14,15] and stripe beam propagating in a bulk crystal $[16,17]$. 2DMI studies have been carried successfully in spatio-temporal configuration $[18,19]$, i.e., when the couple of transverse dimensions is space and time but purely spatial 2DMI has only been evocated briefly in the conference report [20] in a parametric amplification scheme and immediately applied to pattern propagation [21].

In this paper, spontaneous spatial 2DMI is demonstrated in a second harmonic generation ( $\mathrm{SHG}$ ) setup as it was made for the stripe

\footnotetext{
* Corresponding author. Service OPERA-photonique, CP194/5, Université Libre de Bruxelles U.L.B., Avenue F.D. Roosevelt, 1050 Bruxelles, Belgium. Tel.: +32 2650 3203; fax: +322650 4496 .

E-mail address: Michael.Delque@ulb.ac.be (M. Delqué).
}

beam [13], which ensures in addition accurate measurements of the 2DMI spectrum for the first time to our knowledge in $\chi^{(2)}$ medium. Moreover, numerical simulations qualitatively and quantitatively confirm the experimental results. The resulting dynamics can be described by 2 regimes corresponding to amplification of low and high spatial frequency modulations.

\section{Experiments}

The experimental setup is depicted in Fig. 1. $1064 \mathrm{~nm}, 50 \mathrm{ps}, \mathrm{mJ}$ pulses emitted by a Nd:YAG Q-switched mode-locked laser are spatially filtered and scaled to $\mathrm{a} \approx 500 \mu \mathrm{m}$ full-width half-maximum (FWHM) 2D Gaussian intensity profile. Power could be tuned thanks to a couple of polarizers and a half-wave plate. Linear polarization is set to $45^{\circ}$ via a second half-wave plate to reach maximum SHG efficiency in a $2 \mathrm{~cm}$-long KTP crystal cut for type II phase matching. The crystal is oriented in the perfect phase-matching angle (no detuning) in all the experiments in order to optimize the nonlinear efficiency. Fundamental and second harmonic fields (FF and SH, respectively) are separated thanks to a dichroic mirror. Two imaging configurations could be used. On the one hand it was possible to measure near and far field of one single pulse at one wavelength (FF or $\mathrm{SH}$ only). On the other hand FF and $\mathrm{SH}$ of one single pulse could be imaged simultaneously (near or far field only). 2D spatial dynamics will be observable, contrary to what is possible using a stripe beam as in ref. [13] where the instability is limited to one spatial direction (quasi 1D configuration). Our experimental setup gives access to the spatial and spectral features of a 2D spontaneous instability growth. Experimental measurements are presented in Figs. 2-5. For the sake of simplicity, we chose not to represent FF fields as their properties are roughly the same as $\mathrm{SH}$ ones. 


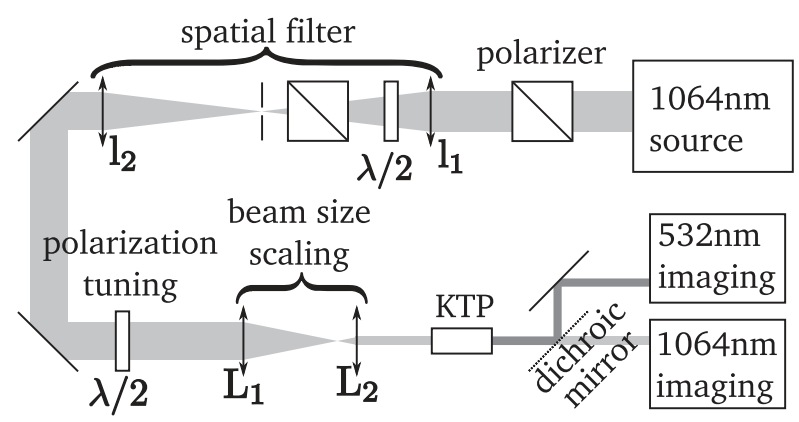

Fig. 1. Experimental setup. $\mathrm{l}_{1}$ and $\mathrm{l}_{2}$ focal lengths equal $1 \mathrm{~m}$. $\left(\mathrm{L}_{1}\right.$, and $\left.\mathrm{L}_{2}\right)$ couple results in a collimated $\approx 500 \mu \mathrm{m}$-wide beam. The KTP crystal was chosen uncoated to avoid damage. No KTP damage has been observed.

Fig. 2(a and b) shows the output near and far fields of SH for a relatively low peak power ( $400 \mathrm{~kW}$ peak power). The FF roughly presents the same 2D Gaussian profile and corresponding spectrum and profile (not shown here). In this case, FF is neither depleted nor deformed, as expected in the weak SHG regime.

For a higher power (1.8 MW peak power, Fig. 2(c and d)), FF and $\mathrm{SH}$ undergo large energy exchanges leading to FF and $\mathrm{SH}$ successive depletions and this regime will be referred to as the depletion regime in the following. The Gaussian nature of the input beam results in a non-homogeneous SHG efficiency which results in a wide ring shape in the SH profile, as shown on Fig. 2(c). Note that FF exhibits the complementary profile, i.e., where SH is intense on Fig. 2(c) FF is depleted and vice-versa, as described in ref. [19] as a preliminary step of SHG MI in the spatio-temporal domain. ${ }^{1}$ This behavior naturally manifests itself through a spectral broadening which is visible in Fig. 2(d) where we observe the formation of a low-frequency ring in the SH spectrum (note that the central peak is saturated in this picture).

Fig. 3(a) reveals that for even higher powers (5 MW peak power), dramatic beam breakup occurs, in a way analog to what is observed in the parametric amplification scheme [20]. High frequency modulation results from the spatial modulation instability and leads to the appearance of a soliton pattern in place of the wide ring shape obtained at a lower power. In the following, this regime will be called the MI regime. The bottom inset of Fig. 3(a) shows a spatial profile extracted from the 2D image and corresponds to a typical MI-soliton array. Input power variations in this highpower range results in modification of the soliton array period, as expected for a MI process. Unfortunately, due to large laser power fluctuations we have not been able to perform an accurate and systematic study of the influence of the input intensity on the characteristic period of the MI patterns. The SH spectrum in Fig. 3(b) now exhibits several orders of modulation rings. However, the spatial frequency range of these spectral rings does not correspond to the frequencies that would be expected for the soliton pattern. Indeed, the soliton array plotted for example in the bottom inset of Fig. 3(a) reveals an average period of $p \approx 56 \mu \mathrm{m}$ corresponding to a transverse wavevector $k_{\perp}=2 \pi / p \approx 112 \mathrm{~mm}^{-1}$. This value is well beyond the ring frequencies that are typically observed below $20 \mathrm{~mm}^{-1}$. Moreover, a circularly symmetric spectrum cannot account for a disordered spatial pattern without any symmetry. Therefore, a more detailed analysis of the spectral features of the generated $\mathrm{SH}$ field has been necessary. This study has been supported by a theoretical analysis as well as numerical simulations.

\footnotetext{
1 These rings would not be present if the input condition was the plane wave solution including both FF and SH components (with correct phase and amplitude) and not the fundamental pump only as in our experimental SHG configuration.
}
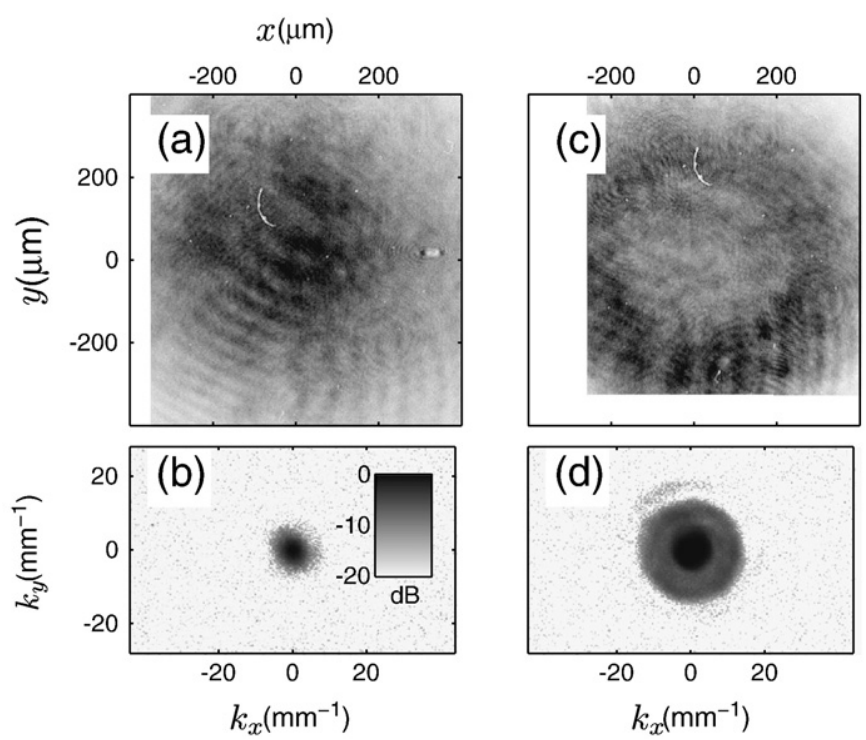

Fig. 2. SH profiles (top) and spatial spectra (bottom, log scale), experiments: (a, b) low power (400 kW peak power) and (c, d) depletion regime (1.8 MW peak power). Grayscale color map will be the same for every spectral image in the article, corresponding to our experimental detection dynamics slightly above $20 \mathrm{~dB}$.

\section{Numerical and analytical investigation}

To get better insight into these 2DMI dynamics, $(2+1)$ D split-step Fourier numerical simulations of light propagation in KTP were performed. The propagation equations corresponding to type II phase matching are those of ref. [1]. Input profiles are $500 \mu \mathrm{m}$-wide 2D Gaussian (intensity FWHM) with $1 \%$ intensity noise with a random phase for FF and quantum noise for SH. Input power is equally divided between ordinary and extraordinary FF components. Type II-cut KTP effective nonlinearity is $d_{\text {eff }}=3.6 \mathrm{pm} / \mathrm{V}$ (from EKSMA optics).

Fig. 3( $c$ and d) shows the numerical results in the high-power MI regime. The soliton pattern is perfectly observable in Fig. 3(c) as well as the expected low-frequency rings in the spectrum in Fig. 3(d). These results are in excellent agreement with the experimental observations. Note that the experimental soliton pattern is roughly
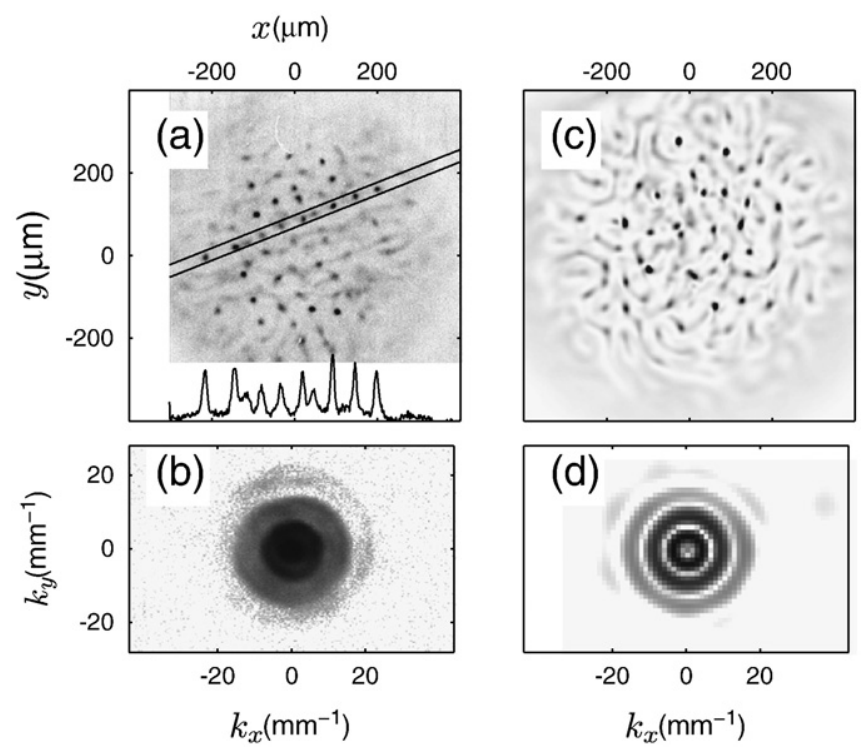

Fig. 3. Experiments: high power $(\approx 5 \mathrm{MW}$ peak power), modulation instability regime, $\mathrm{SH}$ profile (a) and spectrum (b). Bottom inset in (a): profile extracted from the region defined by the couple of black lines. Corresponding simulations (10 MW peak power) in (c) and (d). 

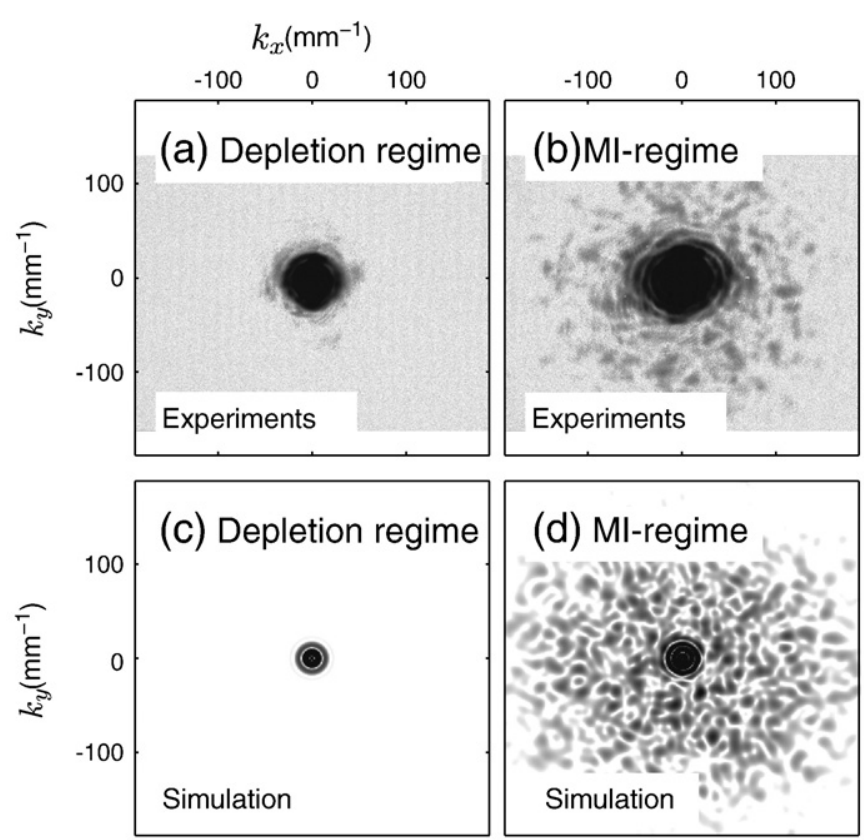

Fig. 4. Highly saturated spatial spectra. Experiments: (a) mid power (1.8 MW peak power), depletion regime and (b) high power (5 MW peak power), modulation instability regime. (c, d): corresponding simulations (2 MW and $10 \mathrm{MW}$ peak power).

spatially stationary from one laser shot to another, which can be explained by the presence of slight permanent defects in the laser beam.

As observed in the experimental results, the low-frequency rings cannot account for the typical size of spatial fluctuations in the soliton pattern as well as for its lack of circular symmetry. To observe the spectral features inherent to the 2DMI regime, we had to look at larger frequencies in the region of the spectrum where the intensity seems to be dominated by noise. In order to observe experimentally this region strongly saturated images of the spatial spectra have been measured. These are shown in Fig. 4(a) and (b) for powers below and above the MI regime, respectively. Corresponding numerical simulations are shown in Fig. 4(c and d). One can now distinguish non
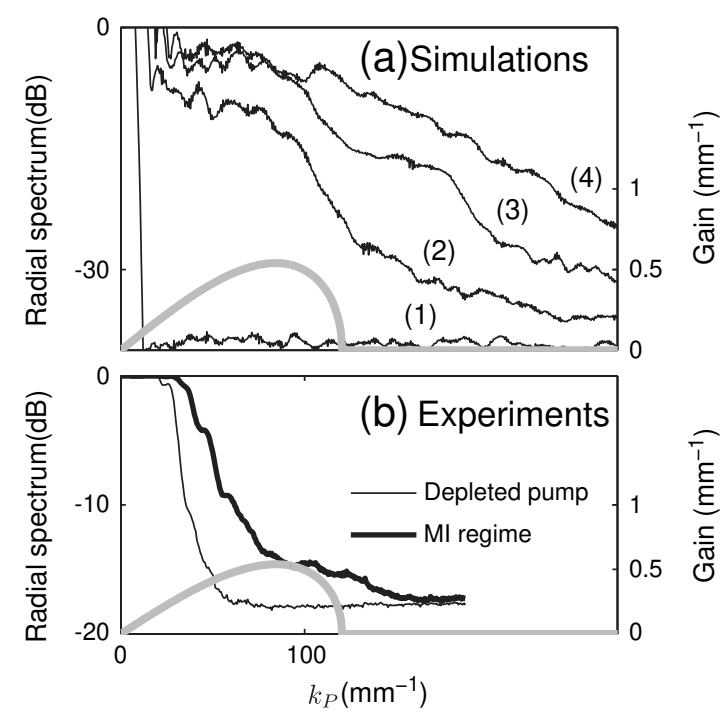

ह

Fig. 5. (a) Radial spectrum evolution during propagation for four steps along the $2 \mathrm{~cm}$ simulated propagation. Spectra (1,2,3, and 4 ) correspond to $2 / 30,15 / 30,20 / 30$ and $30 / 30$ propagation steps, respectively. (b) Experimental output radial spectrum below and above the breakup regime in thin and bold lines, respectively. In both graphs, the analytical MI gain is plotted in a bold gray line. symmetrical structure for the high frequencies. The agreement between numerics and experiment is satisfactory although simulations reveal that the high frequency features inherent to MI may expand further than what could be measured experimentally given the imaging system. Indeed the measure dynamics of the CCDs limits the dynamics of experimental measurements.

The spectra shown in Fig. 5(a) represent the intensity as a function of transverse wavevector modulus. They are extracted from 2D data by averaging the spatial spectra over the polar coordinate of the transverse wavevector plane. The resulting spectra are shown for several propagation steps along the $2 \mathrm{~cm}$ simulated propagation. This representation is used to be able to compare the $2 \mathrm{D}$ results with a simple analytical 1D model derived from ref. [10]. Assuming a circularly symmetry this theoretical stability analysis allows for a quasi 1D derivation, the single dimension being the radial coordinate. The analytical derivation of ref. [10] considers the instability spectrum of a stationary plane wave solution, i.e. a propagation eigenmode including both fundamental and harmonic fields with a locked relative phase. Even if the corresponding initial conditions are not the same as those of the experiment, we chose to use this simple analytical approach because it provides us with a simple means to predict the characteristic MI spectrum.

Spectrum (2) on Fig. 5(a) confirms the initial growth of one limited band starting from the noise background of spectrum (1). Thanks to the analytical model we are able to compute the theoretical band (bold gray curve on Fig. 5) corresponding to the same power level (10 MW total power). The band is characterized by a cut-off frequency around $120 \mathrm{~mm}^{-1}$ which is also observable on spectrum (2) with reasonable agreement. The main difference between the analytical curve and the numerical one is the low-frequency plateau exhibited by the latter one. This plateau corresponds to the previously discussed low-frequency rings.

Spectrum (3) on Fig. 5(a) shows the second-order MI amplification demonstrating the classical cascaded MI process leading to soliton (non-sinusoidal) modulation. Spectrum (4) obtained for full-length propagation shows an even wider spectrum finally corresponding to the soliton pattern. On Fig. 5(b) one can see the experimental output spectra below and above the MI regime extracted from Fig. 4(d) confirming the appearance of the high frequency band in the MI regime only. The agreement with the analytical MI spectral band is of course poorer than that of numerical results. This can be attributed to the pulsed regime of the experiment. The nonlinear propagation at high power introduces temporal dynamics lowering the global measurement quality. However, despite these difficulties, the experimental spectrum clearly exhibits a frequency band in the region predicted by theory.

\section{Conclusion}

In conclusion, spontaneous spatial 2DMI has been experimentally demonstrated in the SHG scheme for the first time to our knowledge and confirmed by $(2+1) \mathrm{D}$ numerical simulations. Spectral features have been observed and interpreted. In particular a non-circularly symmetric structure in the high frequency range has been measured. It was shown to correspond to the breakup of the beam in a soliton pattern and identifies the 2DMI band.

\section{Acknowledgements}

This work is supported by the Interuniversity Attraction Pole program of the Belgian government under Grant IAP6-10 and by a ULB fellowship. This work benefits from the Programme International de Coopération Scientifique PICS-3742 of the French Centre National de la Recherche Scientifique (CNRS). A part of this work was supported by the Groupement de Recherche Photonique Nonlinéaire et Milieux 
Microstructurés GDR-3073 created by CNRS. Computations have been done on the Mesocentre de calcul de Franche-Comté machine.

\section{References}

[1] W.E. Torruellas, Z. Wang, D.J. Hagan, E.W. VanStryland, G.I. Stegeman, L. Torner, C.R. Menyuk, Phys. Rev. Lett. 74 (25) (1995) 5036.

[2] L. Torner, C.R. Menyuk, G.I. Stegeman, Opt. Lett. 19 (20) (1994) 1615.

[3] L. Torner, C.R. Menyuk, W.E. Torruellas, G.I. Stegeman, Opt. Lett. 20 (1) (1995) 13.

[4] A.D. Boardman, K. Xie, A. Sangarpaul, Phys. Rev. A 52 (5) (1995) 4099.

[5] A.V. Buryak, P.D. Trapani, D.V. Skryabin, S. Trillo, Phys. Rep. 370 (2) (2002) 63.

[6] X. Liu, L.J. Qian, F.W. Wise, Phys. Rev. Lett. 82 (23) (1999) 4631.

[7] V.E. Zakharov, A.B. Shabat, Soviet Phys. JETP 34 (1) (1972) 62.

[8] V.I. Bespalov, V.I. Talanov, JETP Lett. 3 (1966) 307.

[9] G.P. Agrawal, Nonlinear Fiber Optics, Optics and Photonics, 3rd Edition, Academic Press, San Diego, 1995.

[10] S. Trillo, P. Ferro, Opt. Lett. 20 (5) (1995) 438.

[11] A. Hasegawa, Opt. Lett. 9 (7) (1984) 288.
[12] H. Maillotte, J. Monneret, A. Barthelemy, C. Froehly, Opt. Commun. 109 (3-4) (1994) 265.

[13] R.A. Fuerst, D.M. Baboiu, B. Lawrence, W.E. Torruellas, G.I. Stegeman, S. Trillo, S Wabnitz, Phys. Rev. Lett. 78 (14) (1997) 2756.

[14] A.D. Boardman, P. Bontemps, K. Xie, J. Opt. Soc. Am. B 14 (11) (1997) 3119

[15] R. Schiek, H. Fang, R. Malendevich, G.I. Stegeman, Phys. Rev. Lett. 86 (20) (2001) 4528.

[16] A. De Rossi, S. Trillo, A.V. Buryak, Y.S. Kivshar, Phys. Rev. E 56 (5) (1997) R4959.

[17] D.M. Baboiu, G.I. Stegeman, Opt. Lett. 23 (1) (1998) 31

[18] X. Liu, K. Beckwitt, F. Wise, Phys. Rev. Lett. 85 (9) (2000) 1871.

19] D. Salerno, S. Minardi, J. Trull, A. Varanavicius, G. Tamosauskas, G. Valiulis, A Dubietis, D. Caironi, S. Trillo, A. Piskarskas, P.D. Trapani, Phys. Rev. Lett. 91 (14) (2003) 143905.

[20] W. Chinaglia, S. Minardi, S. Sapone, G. Coppo, P.D. Trapani, A. Berzanskis, G. Valiulis, K. Staliunas, Nonlinear Guided Waves conference, Opt. Soc. Am. (1999) WA6.

[21] S. Minardi, S. Sapone, W. Chinaglia, P.D. Trapani, A. Beržanskis, Opt. Lett. 25 (5) (2000) 326. 